\title{
Truly stentless autologous pericardial aortic valve replacement: An alternative to standard aortic valve replacement
}

\author{
K. M. John Chan, BMedSci (Hons), BMBS, MSc, FRCS CTh, ${ }^{a}$ Shelley Rahman-Haley, MB BCh, MD, \\ MRCP, ${ }^{\mathrm{b}}$ Tarun K. Mittal, MD, FRCR, ${ }^{\mathrm{c}}$ Jemyrr A. Gavino, BSN, MPH, ${ }^{\mathrm{a}}$ and Gilles D. Dreyfus, MD ${ }^{\mathrm{d}}$
}

Objective: The aim of this study was to determine the feasibility and durability of truly stentless aortic valve replacement using autologous pericardium sutured directly onto the aortic wall.

\begin{abstract}
Methods: Eleven patients (mean age, 55.9 years) requiring aortic valve replacement were recruited. A circular piece of pericardium about $8 \mathrm{~cm}$ in diameter was harvested and treated in $0.6 \%$ glutaraldehyde for 10 minutes. The aortic valve was excised and, with the use of specially designed instruments (CardioMend LLC, Santa Barbara, Calif), the sinotubular junction was sized and the pericardium was tailored to the required size and shape and then sutured directly onto the aortic wall. The reconstructed valve was assessed directly and by echocardiography at the end of the operation; it was assessed by echocardiography and cardiac magnetic resonance imaging at 6 months and yearly. Computed tomographic scan of the aortic valve to assess for valve calcification was performed at last follow-up.
\end{abstract}

Results: Hospital mortality was $0 \%$. Mean follow-up was 6.5 years (range, 5.3-7.5 years). Freedom from structural valve deterioration, thromboembolism, endocarditis and reoperation was $100 \%, 100 \%, 72.7 \%$, and $63.6 \%$, respectively. There were 4 reoperations at $4,13,15$, and 46 months, 3 of them owing to endocarditis and 1 owing to technical failure noted at the time of surgery. The remaining 7 patients are alive and well with a mean New York Heart Association class of 1.3 and normally functioning aortic valves with no calcification.

Conclusions: Truly stentless aortic valve replacement using autologous pericardium sutured directly onto the aortic wall is safe and feasible and has excellent durability up to 7.5 years with no calcification. (J Thorac Cardiovasc Surg 2011;141:276-83)

Aortic valve replacement (AVR) with biological valves is increasingly used in preference to mechanical valves owing to the problems with anticoagulation. However, biological valves are limited in their durability owing to valve degeneration and calcification. ${ }^{1-5}$ The precise mechanism of calcification and pannus formation of bioprosthetic heart valves is not fully known, but immune reaction between the host and implanted valve and mechanical shear stress have been implicated. ${ }^{5-8}$ The use of autologous pericardium for AVR by direct suture of the autologous pericardium to the aortic wall has several potential advantages. First, there is no immune reaction between the host and the implanted

From the Department of Cardiothoracic Surgery, ${ }^{a}$ Royal Brompton and Harefield NHS Foundation Trust, Royal Brompton Hospital, London; the Departments of Cardiology, ${ }^{\mathrm{b}}$ Radiology, ${ }^{\mathrm{c}}$ and Cardiothoracic Surgery, ${ }^{\mathrm{d}}$ Royal Brompton and Harefield NHS Foundation Trust, Harefield Hospital, Middlesex, United Kingdom. Read at the 90th Annual Meeting of The American Association for Thoracic Surgery, Toronto, Ontario, Canada, May 1-5, 2010.

Disclosures: Authors have nothing to disclose with regard to commercial support.

Received for publication May 3, 2010; revisions received Sept 8, 2010; accepted for publication Sept 24, 2010; available ahead of print Oct 21, 2010.

Address for reprints: K. M. John Chan, BMedSci (Hons), BMBS, MSc, FRCS CTh, Department of Cardiothoracic Surgery, 4th Floor, Chelsea Wing, Royal Brompton Hospital, Sydney Street, London SW3 6NP, United Kingdom (E-mail: KMJChan@ btopenworld.com)

0022-5223/\$36.00

Copyright (C) 2011 by The American Association for Thoracic Surgery doi:10.1016/j.jtcvs.2010.09.038 valve and so tissue calcification is reduced. ${ }^{1-3,9}$ Second, direct suture of autologous pericardium onto the aortic wall as valve leaflets to replace the aortic valve eliminates the need for a valve stent, sewing ring, or cuff and so preserves the normal dynamics of the aortic root, which expands in systole and contracts in diastole. ${ }^{10-12}$ As a result, mechanical shear stress on the valve leaflets is reduced, an important cause of leaflet tears and calcification in bioprosthetic valves. ${ }^{1-4,13}$ In addition, the absence of a stent, sewing ring, or cuff in this truly stentless aortic valve maximizes its effective orifice area. These factors may lead to a valve substitute that has optimal hemodynamics and improved durability.

We sought to determine in current contemporary practice the feasibility and durability of truly stentless AVR using autologous pericardium sutured directly onto the aortic wall. We made use of novel specially designed instruments by CardioMend LLC (Santa Barbara, Calif) to size and shape the autologous pericardium. These instruments allowed easy and reproducible sizing and shaping of the autologous pericardium. ${ }^{14}$ To enable application of this procedure in all types of aortic valves, including bicuspid aortic valves, we sutured the autologous pericardium directly onto the aortic wall just above the aortic annulus, and not to the aortic annulus itself. We also assessed valve calcification at late follow-up by performing computed 


\section{Abbreviations and Acronyms \\ $\mathrm{AVR}=$ aortic valve replacement \\ $\mathrm{CPB}=$ cardiopulmonary bypass \\ $\mathrm{CT}=$ computed tomography}

tomographic (CT) scans of the aortic valve. This is the first report of autologous pericardial AVR using this technique and with determination of late valve calcification.

\section{PATIENTS AND METHODS}

The study was approved by the Brompton, Harefield, and National Heart and Lung Institute Research Ethics Committee and was carried out at Harefield Hospital between February 2003 and May 2005. Eleven patients requiring AVR were recruited after written informed consent had been obtained. The mean age was 55.9 years (range, $22-75$ years). There were 8 men and 3 women. The etiology of the aortic valve disease was aortic stenosis in 8 patients ( 3 calcified bicuspid, 5 degenerative), and aortic insufficiency in 3 patients ( 2 endocarditis and 1 congenital quadricuspid valve). Of the 2 patients with endocarditis, 1 had active endocarditis and was operated on during the same hospital admission after receiving 2 weeks of intravenous antibiotics. The other patient had healed endocarditis and was operated on a year after his admission for endocarditis.

\section{Surgical Technique}

All operations were performed by the same surgeon (G.D.) at Harefield Hospital. A circular piece of pericardium about $8 \mathrm{~cm}$ in diameter was harvested. It was cleared of any fibrous deposits and fatty tissue, then immersed in $0.6 \%$ glutaraldehyde for 10 minutes, and then washed in 3 different normal saline washings for a further 10 minutes. During this time, the patient was placed on cardiopulmonary bypass (CPB), an aortic crossclamp applied, a transverse aortotomy performed, antegrade cold blood cardioplegia delivered, the aortic valve excised, and the annulus decalcified.

With the use of specially designed instruments (CardioMend), the sinotubular junction was sized, the pericardium was placed on a base, and a cutting blade of the matching size was placed on top of the pericardium, which cuts it to the required size and shape (a trefoil). The cut pericardium was then mounted on a tissue holder to facilitate suturing it to the aortic wall (Figure 1). The position of the commissures and base of the leaflet cusps on the aortic root was determined using the specially designed instruments and marked with methylene blue. In some cases, for example, in bicuspid aortic valves, the position of the reconstructed commissures was independent of the native commissures. The important principle was to ensure that the newly reconstructed valve was geometrically symmetrical and adequate coaptation of the aortic valve leaflets was achieved.

The prepared autologous pericardium was then sutured directly onto the aortic wall close to the marked annulus using 4-0 Prolene polypropylene sutures (Ethicon, Inc, Somerville, NJ). The 2 commissural sutures for each leaflet were placed first; these went through the aortic wall from inside to outside and the knot was tied on the outside. A double-ended 4-0 Prolene polypropylene suture was then placed at the base of the leaflet cusp; it was stitched to the aortic wall just above the marked annulus and continued on either side toward each commissure. Each running suture starting from the base of the leaflet cusp ended at each commissure where it passed through to the outside of the aorta, at which point the knot was tied (Figure 2). The commissures were then securely fixed by passing another mattress suture from inside the commissure to outside the aorta where it was tied. Leaflet symmetry and coaptation were assessed directly at the end of the procedure before closing the aortotomy and by transesophageal echocardiography on coming off CPB.

\section{Follow-up}

All patients were followed up at the outpatient clinic of the operating surgeon (G.D.) and with echocardiography and cardiac magnetic resonance imaging at 6 weeks, 6 months, and yearly. At last follow-up, al
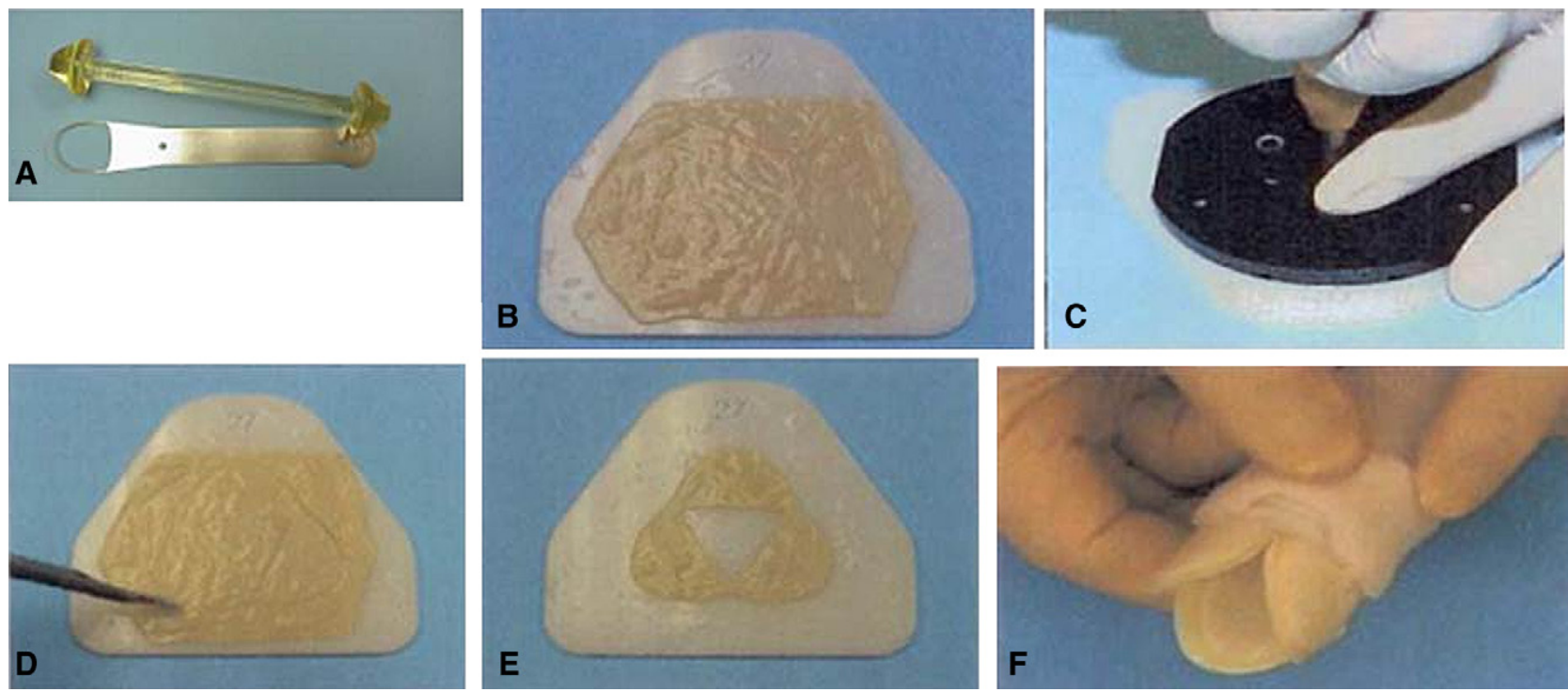

FIGURE 1. Construction of the autologous pericardial aortic valve using CardioMend instruments. A, The sinotubular junction is sized using sizers ranging in size from 19 to $27 \mathrm{~mm}$. B, The harvested autologous pericardium is placed on a base. C, The cutter blade of the appropriate size is placed over the autologous pericardium to cut it to the required size and shape. D, The cut pericardium before removal of the unwanted pieces. Any loose tissue is cut off with a roller cutter. E, The trefoil of cut pericardium is shown after removal of the unwanted pieces. F, The cut pericardium is placed on the tissue holder ready to be sutured to the aortic wall. 

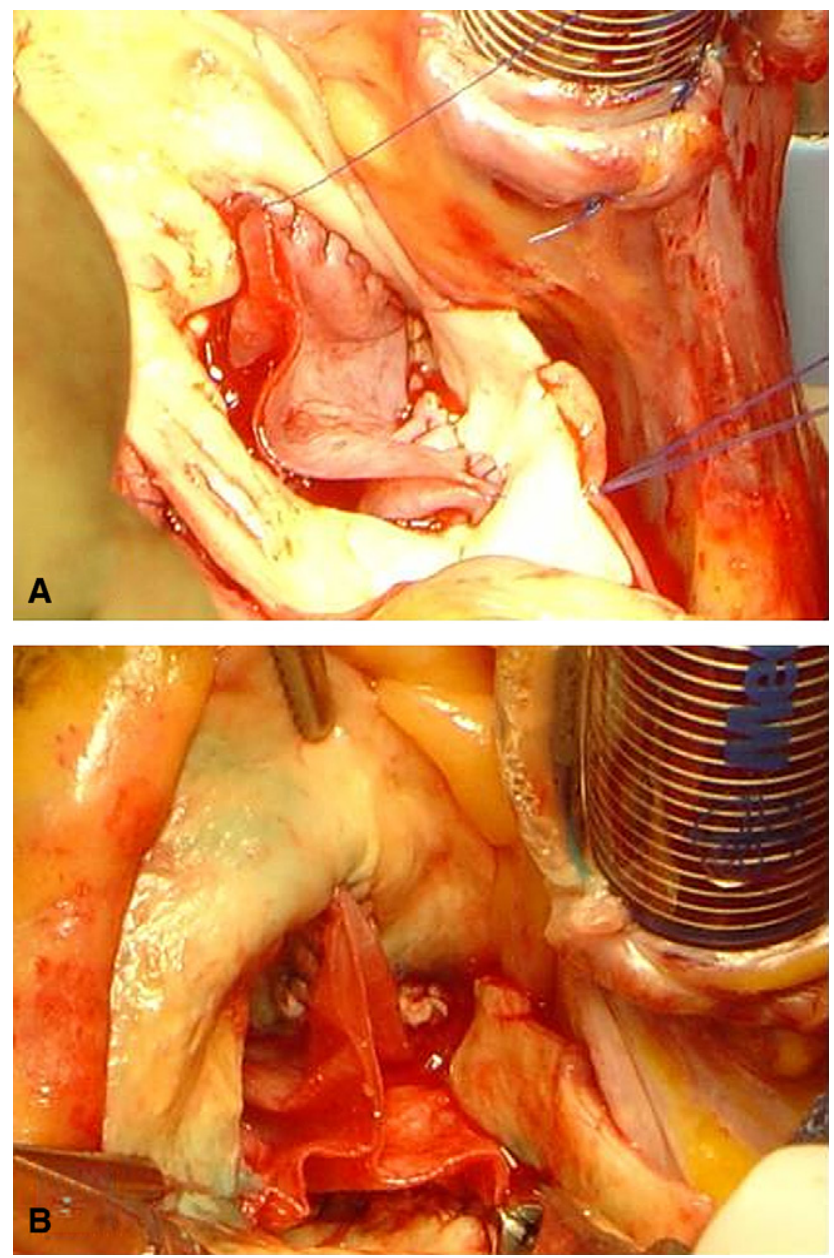

FIGURE 2. Figures showing construction of the new aortic valve using the preshaped autologous pericardium. A, The noncoronary cusp leaflet has been sewn in using continuous 4-0 Prolene polypropylene sutures. The commissural sutures are shown, which pass through the wall of the aorta from inside to outside where the suture is tied. B, All 3 aortic leaflets have been sutured in. The coaptation between the valve leaflets is shown and the symmetry of the reconstructed valve leaflets is demonstrated.

patients were invited to have a CT scan of the aortic valve to assess valve calcification.

\section{Statistical Analysis}

Data are presented as mean \pm standard deviation. Comparison between the baseline and last follow-up data was performed with a paired Student $t$ test using Stata version 10.0 (Stata Corporation, College Station, Tex).

\section{RESULTS}

Hospital mortality was $0 \%$. Concomitant procedures included 1 ascending aortic replacement, 1 resection of subvalvular septal hypertrophy, 1 mitral valve annuloplasty, and 1 coronary artery bypass graft operation. The mean $\mathrm{CPB}$ and aortic crossclamp times were $162.4 \pm 30.6$ minutes and $135.8 \pm 29.1$ minutes, respectively. In patients who did not have concomitant procedures, the mean $\mathrm{CPB}$ and aortic crossclamp times were $140.8 \pm 14.4$ minutes and $114 \pm 9.5$ minutes, respectively. These times compared with mean CPB and aortic crossclamp times of $76.1 \pm 15.5$ minutes and $57.6 \pm 16.6$ minutes, respectively, in patients having isolated conventional stented AVR.

The mean size of the aortic annulus was $26.1 \mathrm{~mm}$ (range, 23-27 mm). The aortic valve was successfully reconstructed in all 11 patients and postoperative transesophageal echocardiography showed excellent symmetry and coaptation of the aortic valve leaflets with no aortic regurgitation in 10 of the 11 patients. In the remaining patient, some prolapse of the right coronary cusp was noted and there was mild aortic regurgitation. All patients made an uneventful postoperative recovery. The mean length of hospital stay was $8.9 \pm 3.2$ days.

There were 4 reoperations: 3 owing to endocarditis at 4 , 13, and 46 months, and 1 at 15 months in the patient who was noted to have some prolapse of the right coronary cusp at the time of the initial operation. Of the 3 in whom endocarditis developed, 2 had endocarditis at their initial operation and the third had endocarditis develop after a dental procedure. The first patient, who was reoperated on at 4 months, had active Staphylococcus aureus endocarditis at his first operation, at which an abscess cavity was present with perforation of the right coronary cusp. He returned with recurrent endocarditis and a tear in the right coronary cusp causing severe aortic regurgitation and underwent valve replacement with a mechanical aortic valve. The second patient, who was reoperated on at 13 months, had Staphylococcus aureus endocarditis 1 year before his original operation. He returned at 13 months with recurrent Staphylococcus endocarditis causing an abscess in the noncoronary sinus and severe aortic regurgitation and underwent a homograft root replacement. The third patient, who was reoperated on at 46 months, had Streptococcus mitis endocarditis develop after a dental procedure, causing a tear in the right coronary cusp and severe aortic regurgitation, and underwent valve replacement with a mechanical aortic valve. Histologic examination of these 3 explanted valves confirmed features of endocarditis with no calcification of the tissue. The fourth patient, who was reoperated on at 15 months, had a technical failure at his original operation. The annulus was asymmetrical with the commissure between the right and noncoronary cusps significantly displaced. This was plicated with 5 Ethibond sutures (Ethicon) reducing the size of the annulus. After construction of the autologous pericardial aortic valve, some prolapse of the right coronary cusp was noted. Transesophageal echocardiography at the end of the procedure showed only mild aortic insufficiency, but this subsequently progressed and necessitated reoperation. Histologic examination of this explanted valve showed preservation of the normal collagen structure of the autologous pericardium with no calcification (Figure 3). 


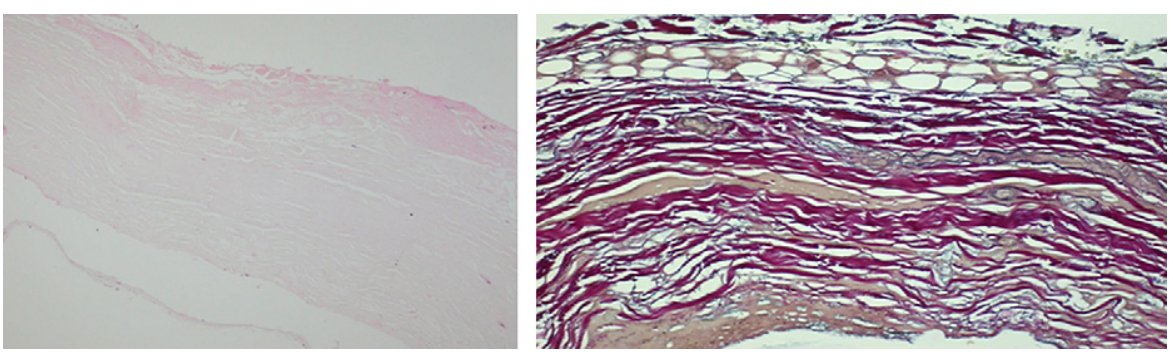

FIGURE 3. Histologic examination of the autologous pericardial valve explanted at 15 months owing to a technical failure showing preservation of the normal collagen structure of the autologous pericardium with no calcification.

The remaining 7 patients are all alive and well. Follow-up was $100 \%$ complete with a mean duration of 6.5 years (range, 5.3-7.5 years). Mean New York Heart Association class was 1.3 , and all had normally functioning aortic valves with a mean peak gradient of $10.9 \mathrm{~mm} \mathrm{Hg}$, mean peak velocity of $1.4 \mathrm{~m} / \mathrm{s}$, and mean aortic insufficiency grade of 0.3 (Table 1). No patient had greater than mild aortic regurgitation or stenosis. The normal function of the reconstructed aortic valve was confirmed by cardiac magnetic resonance imaging in all patients (Figure 4). Five patients consented to a CT scan of the aortic valve at a mean of 6.5 years after surgery (range, 6.0-7.2 years). In all 5 patients, there was no calcification of the aortic valve. Freedom from structural valve deterioration, thromboembolism, endocarditis, and reoperation was $100 \%, 100 \%, 72.7 \%$, and $63.6 \%$, respectively.

\section{DISCUSSION}

This prospective pilot study of 11 patients has demonstrated that replacement of the aortic valve with autologous pericardium sutured directly onto the aortic wall is safe and feasible. The hemodynamic performance of the valve is excellent with a minimal gradient across it and no aortic regurgitation. The absence of a supporting stent and sewing ring in this truly stentless aortic valve maximizes its effective orifice area and may make it particularly useful in those with small aortic roots or poor left ventricular function, although

TABLE 1. Comparison of baseline and last follow-up clinical and echocardiographic data

\begin{tabular}{lrccl}
\hline \multicolumn{1}{c}{ Parameters } & Baseline & Follow-up & Change & $\boldsymbol{P}^{*}$ \\
\hline NYHA class & $2.4(0.5)$ & $1.3(0.8)$ & -1.1 & .0047 \\
LVESD $(\mathrm{mm})$ & $26.7(2.9)$ & $26.6(4.0)$ & -0.1 & .94 \\
LVEDD $(\mathrm{mm})$ & $44.1(2.0)$ & $43.9(2.9)$ & -0.2 & .82 \\
Peak aortic valve & & $10.9(10.5)$ & & \\
$\quad$ gradient $(\mathrm{mm} \mathrm{Hg})$ & & & & \\
Peak aortic valve & & $1.4(0.8)$ & & \\
$\quad$ velocity $(\mathrm{m} / \mathrm{s})$ & & & & \\
Mean aortic regurgitation & & $0.3(0.5)$ & &
\end{tabular}

grade (0-4)

\begin{tabular}{lllll} 
Left ventricular mass $(\mathrm{g})$ & $234(52.1)$ & $195.1(41.1)$ & -38.9 & .068 \\
\hline
\end{tabular}

NYHA, New York Heart Association; LVESD, left ventricular end-systolic dimension; LVEDD, left ventricular end-diastolic dimension. *Student paired $t$ test. its use in the latter must be balanced with the increased CPB time required for this procedure. ${ }^{15}$ It has excellent durability up to 7.5 years with a freedom from structural valve deterioration of $100 \%$, which is comparable with, if not better than, conventional bioprosthetic aortic valves given the younger age group of patients in this study. ${ }^{1,2,16}$ This is consistent with a previous study that reported a freedom from structural valve deterioration of $80 \%$ at 10 years in young patients who had autologous pericardial AVR. ${ }^{17}$ The autologous pericardial aortic valve is nonthrombogenic with no need for anticoagulation as demonstrated in this and previous studies. ${ }^{17}$

The use of autologous pericardium avoids any immune reaction between the host and the implanted heart valve and so minimizes tissue calcification and pannus formation, which are important causes of structural valve deterioration in bioprosthetic heart valves. ${ }^{1,3,5,8}$ This was confirmed in the present study by the absence of any valve calcification when assessed by CT scan at a mean of 6.5 years and also by the absence of any calcification in the 4 explanted autologous pericardial valves. ${ }^{3,9,17,18}$

Previous attempts at using untreated autologous tissue for AVR had been troubled by shrinkage and retraction of the tissue. ${ }^{19}$ This problem appears to have been alleviated by pretreatment of the autologous tissue with $0.6 \%$ glutaraldehyde, which preserves the structure of the autologous tissue, a concept first described in $1986 .{ }^{20}$ A recent study demonstrated freedom from structural valve deterioration of $80 \%$ at 10 years with autologous pericardial aortic valves pretreated with glutaraldehyde and no calcification or tissue retraction in those who had reoperations. ${ }^{17}$ Studies of aortic valve repair with leaflet augmentation using autologous pericardium pretreated with glutaraldehyde have also consistently reported excellent durability of the autologous pericardial patch, with 1 study reporting no structural dysfunction of the autologous pericardial leaflet extension at 13 years, and in patients needing reoperation, no calcification was found. ${ }^{21}$ Similarly, glutaraldehyde-treated autologous pericardial patches used for leaflet extension in mitral valve repair have shown excellent durability with no calcification. ${ }^{22}$

The direct suture of autologous pericardium to the aortic wall creating a new aortic valve avoids the need for any 

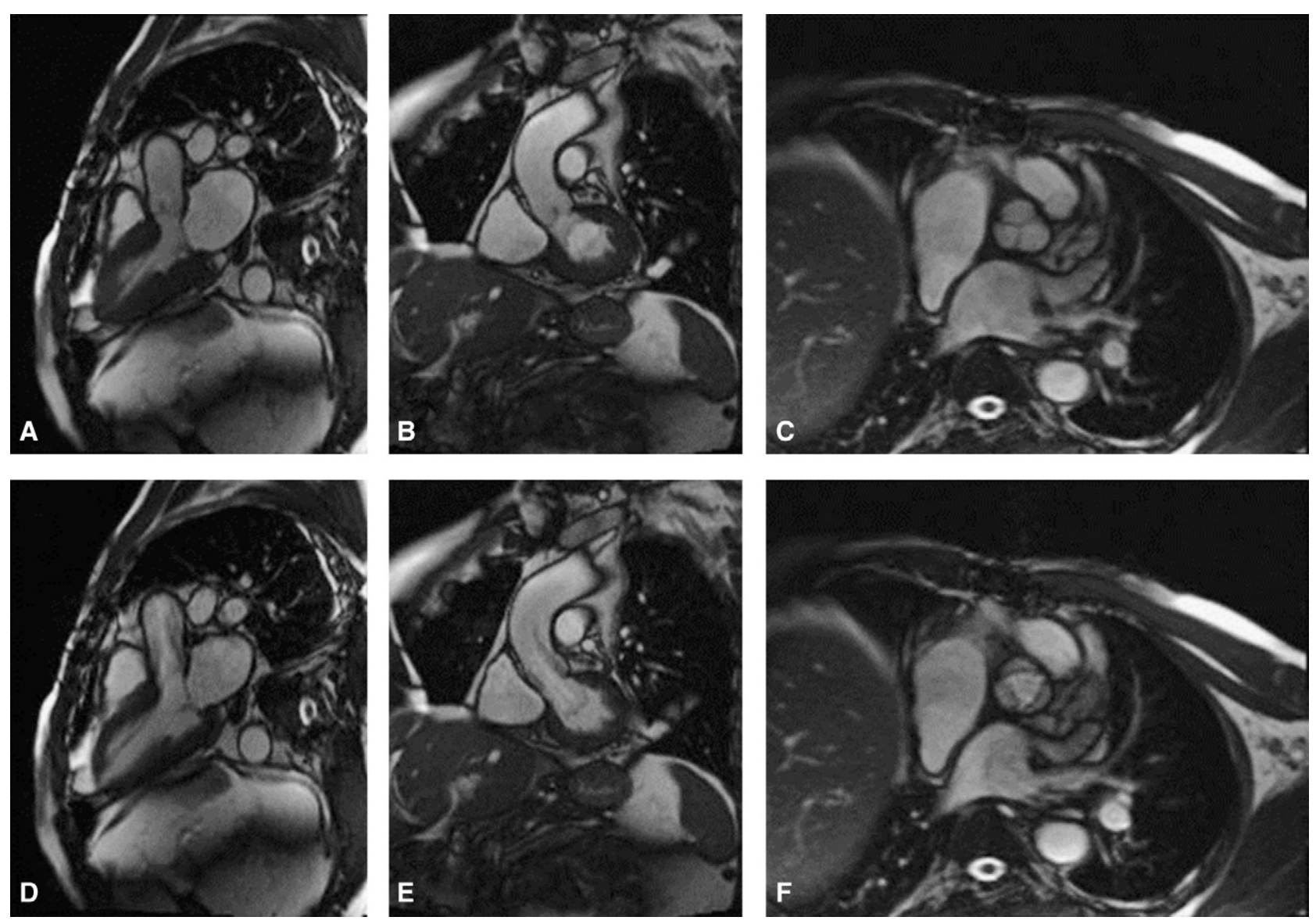

FIGURE 4. Series of cardiac magnetic resonance images demonstrating the normal function and geometry of the reconstructed autologous pericardial aortic valve in a patient 6 years after the operation. A and B, Left ventricular outflow tract and ascending aortic views in diastole demonstrating normal coaptation between the valve leaflets and no regurgitation. C, Transverse aortic valve view in diastole demonstrating coaptation between the valve leaflets and its symmetrical trileaflet geometry. D, E, and F, Corresponding images in systole showing normal symmetrical opening of the reconstructed aortic valve leaflets.

supporting stent, sewing ring, or cuff and so preserves the normal dynamics of the aortic root, which expands in systole and contracts in diastole. ${ }^{10-12}$ This reduces the mechanical shear stress on the reconstructed valve leaflet as it is allowed to move with the aortic wall during the cardiac cycle and so may improve its durability by reducing leaflet tears and also calcification.,13,18 Valve hemodynamics and effective orifice area are also optimized. Studies on stentless aortic valve bioprostheses have shown this to be advantageous, particularly in patients with small aortic roots and impaired left ventricular function. ${ }^{15}$ The benefits from this truly stentless valve may be even better than those of commercially available stentless valves, which all have a sewing cuff and thus do not fully allow for preservation of the normal dynamics of the aortic root, although the reproducibility of this procedure among different surgeons may not be as good as in commercially available stentless valves.

In a previous study of autologous pericardial AVR, the autologous pericardium was sutured directly to the aortic annulus, not the aortic wall. ${ }^{17}$ This has important limita- tions inasmuch as it assumes that all 3 aortic sinuses are symmetrical and would exclude bicuspid aortic valves from the procedure. The sizing and shaping of the autologous pericardium also did not appear easily reproducible. In the present study, the autologous pericardium was sutured directly to the aortic wall just above the aortic annulus (and not to the aortic annulus itself), allowing us to reconstruct 3 symmetrical aortic valve leaflets independent of the geometry of the native aortic valve. Three of the surgically treated patients had bicuspid aortic valves and 1 had a quadricuspid aortic valve. We made use of specially designed instruments (CardioMend), which allowed for easy and reproducible sizing and shaping of the autologous pericardial aortic valve. ${ }^{14}$ Although a cost-comparison analysis was not done, it is likely that this procedure will have a significantly lower cost than that of conventional AVR using a bioprosthesis.

It is accepted that the implantation of autologous pericardial aortic valves by direct suture to the aortic wall is more technically challenging than a conventional stented or 
stentless bioprosthetic valve, particularly when there is asymmetry of the aortic annulus. It can be challenging to determine the true height of the commissures, and some experience with aortic valve surgery is necessary. The important principle is to create 3 symmetrical valve leaflets and commissures and to ensure adequate coaptation between them with no prolapse at the end of the procedure. Alignment of the 3 new commissures of the reconstructed valve may need to be independent of that of the native valve if asymmetry is present, and this is enabled by suturing the new valve directly to the aortic wall just above the native aortic annulus and not to the aortic annulus itself. In the present study, technical failure resulted in the need for reoperation in 1 patient. It should be noted that the present study reports the first 11 patients operated on by the same surgeon (G.D.) using this new technique and so is part of the learning curve of the surgeon. It is likely that the results of subsequent patients will be better once the learning curve has been passed.

The high rate of endocarditis of $27.3 \%$ at 7.5 years is a concern and appears much higher than that reported for bioprosthetic aortic valves. ${ }^{1,2}$ A previous study using autologous pericardium for AVR also reported a high rate of endocarditis of $10.7 \%$ at 16 years. ${ }^{17}$ It is unknown whether autologous tissue has an inherently higher risk of endocarditis. It should be noted, however, that in the present study, 2 of the 3 patients in whom endocarditis developed had endocarditis as the indication for their original operation, and reinfection of prosthetic valves after valve replacement for endocarditis is a recognized problem. The third patient in whom endocarditis developed in the present study had an invasive dental procedure. A further factor that should be considered is that ultrafiltration of the glutaraldehyde to limit bacterial contamination was not done in this study, and preparation of the chemical was not done in the operating theater but in the pharmacy department, where conditions of asepsis may not have been as vigilant. Nonetheless, the results of the present study strongly suggest that autologous tissue should not be used for valve replacement in the presence of endocarditis, and appropriate antibiotic prophylaxis needs to be taken for any invasive dental or surgical procedure.

This truly stentless autologous pericardial aortic valve with optimal hemodynamics and possibly improved durability may be particularly advantageous in younger active patients who either cannot or do not want to take anticoagulation. It is much less invasive and requires less distortion of the aortic root geometry than does a Ross procedure or a homograft. Although it was previously believed that the pulmonary autograft was the ideal valve substitute in these patients, it is now emerging that its durability may be limited beyond the first decade, in addition to the previously recognized limited durability of the homograft in the pulmonary position. ${ }^{23}$ It is also a more technically challenging operation to perform. Homografts in the aortic position, although previously used in younger patients, have now been shown to be less durable than commercially available stentless biological valves and so cannot be recommended as the ideal valve substitute in these patients. ${ }^{24}$

A further advantage in this truly stentless autologous pericardial aortic valve is that should reoperation be necessary, it is technically a much easier operation to perform than a reoperation for a failed homograft or bioprosthetic valve; in fact, it is like a first-time AVR once access to the aortic valve has been obtained. There is no calcification of the valve leaflets or the aortic wall, as would be the case if a homograft had been used, no need to explant a bioprosthetic valve, and no need to reconstruct the aortic root, as may be necessary if a stentless valve or homograft had been implanted as a root replacement. Furthermore, should transcatheter valve implantation be considered for these patients in future, there is no concern of patient-prosthesis mismatch as may occur if a transcatheter valve is placed inside a stented or stentless bioprosthesis.

\section{CONCLUSIONS}

Truly stentless AVR using autologous pericardium sutured directly onto the aortic wall without supporting stents is safe and feasible and results in a valve with excellent hemodynamics and preservation of the normal dynamics of the aortic root. It has excellent durability up to 7.5 years with no calcification. This valve may be particularly useful for younger patients who cannot or do not want to take anticoagulation and for those with a small aortic root and poor left ventricular function. Reoperation, if necessary, is technically unchallenging compared with other valve substitutes. The overall experience with autologous pericardial aortic valves is still limited and further studies involving larger numbers of patients are needed, including randomized trials comparing autologous valves with conventional bioprosthetic aortic valves.

Winston Banya, MSc, Statistician at the Royal Brompton Hospital, London, United Kingdom, provided statistical analysis of the data. Jack Love, MD, DPhil, Chief Executive Officer and Chief Scientific Officer of CardioMend LLC, provided helpful comments, the images in Figure 1, and the instruments for sizing and cutting the autologous pericardium.

\section{References}

1. Aupart MR, Sirinelli AL, Diemont FF, Meurisse YA, Dreyfus XB, Marchand MA. The last generation of pericardial valves in the aortic position ten year follow-up in 589 patients. Ann Thorac Surg. 1996;61:615-20.

2. Banbury MK, Cosgrove DM, Lytle BW, Smedira NG, Sabik JF, Saunders CR. Long-term results of the Carpentier-Edwards pericardial aortic valve: a 12 year follow-up. Ann Thorac Surg. 1998;66:S73-6.

3. Grabenwoger M, Fitzal F, Gross C, Hutschala D, Bock P, Brucke P, et al. Different modes of degeneration in autologous and heterologous heart valve prostheses. J Heart Valve Dis. 2000;9:104-9. 
4. Mohammadi S, Baillot R, Voisine P, Mathieu P, Francois D. Structural deterioration of the Freestyle aortic valve: mode of presentation and mechanisms. J Thorac Cardiovasc Surg. 2006;132:401-6.

5. Butany J, Zhou T, Leong SW, Cunningham KS, Thangaroopan M, Jeggatheeswaran A, et al. Inflammation and infection in nine surgically explanted Medtronic Freestyle stentless aortic valves. Cardiovasc Pathol. 2007; $16: 258-67$

6. Human P, Zilla P. The possible role of immune responses in bioprosthetic heart valve failure. J Heart Valve Dis. 2001;10:460-6.

7. Konakci K, Bohle B, Blumer R. Alpha-Gal on bioprosthesis: xenograft immune response in cardiac surgery. Eur J Clin Invest. 2005;35:17-23.

8. Siddiqui RF, Abraham JR, Butany J. Bioprosthetic heart valves: modes of failure. Histopathology. 2009;55:135-44.

9. Duran CMG, Gometza B, Kumar N, Gallo R, Martin-Duran R. Aortic valve replacement with freehand autologous pericardium. J Thorac Cardiovasc Surg. 1995;110:511-6.

10. Cheng A, Dagum P, Miller DG. Aortic root dynamics and surgery: from craft to science. Philos Trans R Soc Lond B Biol Sci. 2007;362:1407-19.

11. Rodriguez F, Green G, Dagum P, Nistral JF, Harrington KB, Daughters GT, et al. Left ventricular volume shifts and aortic root expansion during isovolumic contraction. J Heart Valve Dis. 2006;15:465-73.

12. Lansac E, Lim HS, Shomura Y, Lim KH, Rice NT, Goetz W, et al. A fourdimensional study of aortic root dynamics. Eur J Cardiothorac Surg. 2002;22: 497-503.

13. Thubrikar MJ, Deck JD, Aouad J, Nolan SP. Role of mechanical shear stress in calcification of aortic bioprosthetic valves. J Thorac Cardiovasc Surg. 1983;86: 115-25.

14. Hanlon JG, Suggit RW, Gibbs E, McMeeking RM, Love JW. Geometric optimization of a tissue pattern for semilunar valve reconstruction. $J$ Heart Valve Dis. 1999;8:609-13.

15. Gulbins $H$, Reichenspurner $H$. Which patients benefit from stentless aortic valve replacement? Ann Thorac Surg. 2009;88:2061-8.

16. McClure RS, Narayanasamy N, Wiegerinck E, Lipsitz S, Maloney A, Byrne JG, et al. Late outcomes for aortic valve replacement with the Carpentier-Edwards pericardial bioprosthesis: up to 17 year follow-up in 1000 patients. Ann Thorac Surg. 2010;89:1410-6.

17. Al Halees Z, Al Shahid M, Al Sanei A, Sallehuddin A, Duran C. Up to 16 years follow-up of aortic valve reconstruction with pericardium: a stentless readily available cheap valve? Eur J Cardiothorac Surg. 2005;28:200-5.

18. Gross C, Simon P, Grabenwoger M, Mair R, Sihorsch K, Kypta A, et al. Mid term results after aortic valve replacement with the autologous tissue cardiac valve. Eur J Cardiothorac Surg. 1999;16:533-9.

19. Dalichau H, Gonzalez-Lavin L, Ross DN. Autologous fascia lata transplantation for heart valve replacement. Thorax. 1972;27:18-22.

20. Love JW, Calvin JH, Phelan RF, Love CS. Rapid intraoperative fabrication of an autogenous tissue heart valve: a new technique. Proceedings of the third international symposium on biologic and bioprosthetic valves: Yorke Medical Books; 1986. p. 691-8.

21. Jeong DS, Kim K-H, Ahn H. Long-term results of the leaflet extension technique in aortic regurgitation: thirteen years of experience in a single centre. Ann Thorac Surg. 2009;88:83-9.

22. Chauvand S, Jebara V, Chachques JC, el Asmar B, Mihaileanu S, Perier P, et al. Valve extension with glutaraldehyde-preserved autologous pericardium: results in mitral valve repair. J Thorac Cardiovasc Surg. 1991;102:171-7.

23. Takkenberg JJM, Klieverik LMA, Schoof PH, van Suylen R-J, van Herwerden LA, Zondervan PE, et al. The Ross procedure. A systematic review and meta-analysis. Circulation. 2009;119:222-8.

24. El-Hamamsy I, Clark L, Stevens LM, Sarang Z, Melina G, Takkenberg JJM, et al. Late outcomes following Freestyle versus homograft aortic root replacement. Results from a prospective randomized trial. J Am Coll Cardiol. 2010; 55:368-76

\section{Discussion}

Dr Michael Acker (Philadelphia, $\mathrm{Pa}$ ). I have a couple of technical questions. Can this procedure be done in a bicuspid valve that might not be symmetric where you have to really nail where your commissures are, and what are the size limitations of this technique?
Dr Chan. Thank you for your question, Dr Acker. Yes, it can be done. We have in fact done a few bicuspid valves and even a quadricuspid valve. The important principle is to attain symmetry of the leaflets and commissures at the end of the operation, and this is done in some cases independent of the native commissures. We place the sutures not actually on the annulus itself but slightly above the annulus on the aortic wall.

The sizes provided by the company (CardioMend) that supplies these instruments range from $19 \mathrm{~mm}$ to $27 \mathrm{~mm}$, and we have done operations in all these sizes.

Dr Nawwar Al-Attar (Paris, France). My question is practically a follow-up of the same question. You had described 1 of your patients as having had a quadricuspid valve. Was this a patient who had a technical failure? Would this procedure be contraindicated for patients who do not have tricuspid valves, for your kind of valve, for this technique?

Dr Chan. Thank you for your question. The patient who had a quadricuspid valve is doing very well. The complication was in a patient with a bicuspid valve who had a very gross asymmetry of the annulus and commissures. It is certainly much easier to do this operation in a tricuspid valve with symmetry of the annulus, and it is certainly more challenging in bicuspid valves; in quadricuspid valves it is certainly very challenging.

Dr Wolfgang Harringer (Braunschweig, Germany). What we have learned from the trials with intraoperatively manufactured stented valves is that the quality of the pericardium is not equal in all the patients. In other words, it differs depending on the area of the pericardium you are taking; it also differs, for example, between a strong man and an old woman. Did you perform any quality assessment? What guided your judgment to choosing appropriate areas of the pericardium?

Dr Chan. Thank you for your question. No, there was no precise quality control. It was macroscopic on surgical view. The pericardium had to be clean. We cleaned it of all fatty and fibrous tissue, and it had to look good macroscopically. But we did not do any further analysis on this pericardium. It was autologous and harvested at the time of surgery.

Dr Boulos Asfour (Sankt Augustin, Germany). We have done about 120 repairs in aortic valves in all kind of sizes, even in infants. Many times when you look at the valve, you do not want to throw away the whole valve and put in this kind of pericardial valve. In the case of a valve affected by endocarditis with 1 leaflet being severely damaged, would you consider cutting out one of these cusps, replacing it, and leaving the others alone?

Dr Chan. Thank you for your question. Yes, the option of aortic valve repair is certainly open. In this series, none of the patients would have been unsuitable for aortic valve repair; 8 of them had calcified degenerative valve disease and the other 2 had severe aortic regurgitation from endocarditis. We are a bit concerned about using this for endocarditis now, given the high recurrence of endocarditis in our series and also in Duran's article. Thus we would not recommend this technique in the presence of endocarditis today.

Dr A. W. Atkinson (Raleigh, NC). This refers to the endocarditis question. You mentioned an earlier article with a high incidence of late endocarditis. It appears that this new biological system, if you want to call it that, is not inert, or there is a chronic 
inflammatory process that is the nidus for endocarditis. Can you discuss that? Did you learn anything from the explantation of the infected valve that you had?

Dr Chan. Thank you for your question. I do not think we know the answer. In our series all 3 patients in whom endocarditis devel- oped had risk factors to account for the endocarditis. Two of them had Staphylococcus aureus endocarditis as the indication for the original operation and a third had a dental procedure before endocarditis developed. However, I think the incidence of endocarditis is certainly a concern. 